\author{
¿Cómo citar este artículo? \\ Marulanda-Valencia, F. A., y Restrepo-Montes, J. A. (septiembre-diciembre, 2020). Estrategias para \\ fortalecer la ocupación hotelera: estudio de caso en una Cadena de la Ciudad de Medellín. Revista \\ Virtual Universidad Católica del Norte, (61), 183-202. https://www.doi.org/10.35575/rvucn.n61a11
}

\title{
Estrategias para fortalecer la ocupación hotelera: estudio de caso en una Cadena de la Ciudad de Medellín'
}

\section{Strategies to strengthen hotel occupancy: case study in a Chain of the City of Medellin}

\section{Flor Ángela Marulanda-Valencia}

Doctora en Ingeniería-Industria y Organizaciones Departamento de Ingeniería de la Organización, Universidad Nacional de Colombia

Medellín, Colombia

famarulan@unal.edu.co

Orcid: https://orcid.org/0000-0003-2036-0639

CVLAC: https://scienti.minciencias.gov.co/cvlac/visua-

lizador/generarCurriculoCv.do?cod_rh $=0001107240$

\section{Jonathan Alexis Restrepo-Montes}

Magister en Ingeniería Administrativa Universidad Nacional de Colombia Medellín, Colombia

joarestrepomo@unal.edu.co

Orcid: https://orcid.org/0000-0003-1852-813X

CVLAC: https://scienti.minciencias.gov.co/cvlac/visualizador/generarCurriculoCv.do?cod_rh=0001769223

Recibido: 16 de marzo de 2020 Evaluado: 17 de mayo de 2020

Aprobado: 11 de agosto de 2020

Tipo de artículo: Investigación Científica y Tecnológica

\section{Resumen}

Los hoteles de Medellín han atravesado dificultades relacionadas con la competencia legal e ilegal, lo que ha impactado de forma negativa sus ventas y rentabilidad. El objetivo de este estudio fue generar estrategias que permitan al sector formal de hospedaje fortalecer la competitividad y por tanto incrementar sus niveles de ocupación. Para ello, se realizó el diagnóstico de una cadena hotelera en Medellín, a partir de fuentes primarias como entrevista con funcionarios de la misma organización y revisión de opiniones de los clientes. Con base en el diagnóstico, se diseñaron algunas estrategias que fueron validadas, utilizando la herramienta de prospectiva estratégica Color Insight. Se destaca el aporte del estudio, en relación con la valoración de los lineamientos de acción propuestos, la cual fue realizada por personas expertas en el sector, con la participación de empleados del área de mercadeo de la cadena, académicos de tres universidades y funcionarios de la Subsecretaría de Turismo y Cotelco. Las estrategias con mayor valoración fueron las relacionadas con la gestión humana, el fortalecimiento de la internacionalización y los procesos internos de la empresa.

Palabras clave: Color Insight; Estrategias; Hotelería; Prospectiva; Turismo.

1 Artículo derivado del trabajo de investigación realizado en el marco de la tesis de maestría "Diseño de estrategias para el fortalecimiento de la competitividad e incremento de los niveles de ocupación de una Cadena Hotelera en la regional Medellín, mediante la definición de escenarios derivados de la prospectiva estratégica", en la Universidad Nacional de Colombia, sede Medellín. 


\section{| Abstract}

Hotels in Medellin have faced difficulties related to legal and illegal competition, which negatively have affected their sales and profitability. The objective of this study was to generate strategies that allow the formal hosting sector to strengthen competitiveness and therefore increase its occupancy levels. For this, the diagnosis of a Hotel Chain in Medellin was carried out from primary sources as an interview with officials of the same Organization and review of customer opinions. Based on the diagnosis, some strategies were designed and validated using the Color Insight strategic foresight tool. The contribution of the study stands out, in relation to the assessment of the proposed action guidelines, which was carried out by experts in the sector, with the participation of employees from the marketing area of the same Organization, academics from three universities and officials from the undersecretary of tourism and Cotelco. The strategies with the highest valuation were those related to human management, strengthening the internationalization, and internal processes of the company.

Palabras clave: Color Insight; strategies; Hospitality; Foresight; tourism.

\section{| Introducción}

El sector del turismo involucra una cantidad variada de subsectores y agentes, entre ellos el de hospedaje, considerado uno de los principales dentro de la actividad turística (Monsalve y Hernández, 2015; González-Posada y Reyes-Bedoya, 2019). Los autores sostienen, además, que la efectiva gestión de los servicios hoteleros, desde un enfoque del desarrollo sostenible, contribuye al progreso y promoción de los destinos.

Al revisar las estadísticas de ocupación hotelera divulgadas por Cotelco, en la Ciudad de Medellín, se nota que esta no incrementa en forma proporcional con el número de visitantes, incluso en algunos de los últimos años ha disminuido. Para los hoteles tradicionales, esta situación se convierte en una fuerte amenaza, ya que, por su estructura de costos y gastos fijos, especialmente los relacionados con la nómina, el mantenimiento de la infraestructura, y los contratos asociados a la tecnología, la disminución en los ingresos puede implicar grandes pérdidas. A diferencia de las empresas asociadas al sector industrial, en las que, si no se vende un producto hoy se podría vender el día de mañana, y a menor precio, en la hotelería, una habitación no vendida por noche representa una pérdida irrecuperable para el negocio.

En general, el turismo es un importante renglón en la economía de los países. Así, en Colombia, junto con el comercio, los restaurantes y hoteles, son los sectores que más empleo generan, con una participación del 27 \% en el ámbito nacional (Departamento Administrativo Nacional de Estadística -DANE-, 2019). Además, el turismo, medido desde la rama de hoteles y restaurantes, representó el 3,78 \% del PIB en Colombia, durante 2017 (Ministerio de Comercio Industria y Turismo - MinCIT-, 2018).

En Medellín, el número de visitantes prácticamente se ha duplicado en el último decenio, al pasar de 2.250.000, en el período 2008-2013, a 4.350.000, aproximadamente, en el período 2014-2019 (Sistema de Indicadores Turísticos de Medellín y Antioquia -SITUR-, 2020); pero también se ha dado un gran incremento de la oferta formal de hospedaje. 


\section{Contexto del subsector de alojamiento}

La construcción de nuevas sedes de las cadenas tradicionales y la entrada de cadenas internacionales empieza a causar el fenómeno que, autores como Matiz et al. (2011) denominan "turbulencia hotelera" (p. 21). Este crecimiento se debe, en gran parte, al Decreto 2755 del año 2003, que ofreció la exención del impuesto sobre la renta para los nuevos hoteles, a partir de ese mismo año. El incentivo se había ofrecido por 30 años, pero en realidad duró hasta el 31 de diciembre de 2017 (Presidencia de la República de Colombia, 2016). De acuerdo con Duque (2018), las inversiones que se hicieron durante el tiempo que duró el incentivo fue de aproximadamente 6 billones de pesos, en la construcción y/o remodelación de 74.000 habitaciones.

Por otro lado, en la Ciudad se ha detectado un fenómeno asociado a la llamada "hotelería paralela", también denominada hotelería informal, que consiste en la prestación de servicios de alojamiento en viviendas dedicadas de forma ocasional al uso turístico, generalmente en apartamentos o habitaciones de edificios residenciales, los cuales no ofrecen un contrato formal de hospedaje ni los beneficios derivados por su acuerdo. Esto se traduce en un detrimento en la calidad y sostenibilidad de los servicios turísticos por la falta de control sobre los establecimientos no formales (De Duque et al., 2010). Los autores sostienen que el impacto económico de esta práctica irregular es significativo en las economías locales; de igual manera, las administraciones públicas dejan de percibir importantes recaudos por impuestos. Muchos de estos hospedajes son ofrecidos por empresas como Airbnb, conocidas como plataformas peer-to-peer, las cuales han tenido un rápido crecimiento por dos factores clave: innovaciones tecnológicas y flexibilidad del lado de la oferta (Zervas et al., 2017). En relación con este aspecto, Moreno-Izquierdo et al. (2020) afirman que la industria del turismo está en constante evolución, tanto en términos de demanda como de oferta, lo que altera significativamente el comportamiento de los empresarios, las autoridades públicas y los usuarios.

Este y otros factores han hecho que la ocupación hotelera presente un comportamiento irregular. En 2014 esta fue del 61.9 \%, subió hasta 69.2 \% en 2016, y en 2018 cayó nuevamente ubicándose en 62.4 \% (Asociación Hotelera y Turística de Colombia, Cotelco, 2018). Este comportamiento genera subutilización de la capacidad instalada, para el sector formal de alojamiento, el cual, en Medellín, ha realizado grandes esfuerzos encaminados a la prestación de un mejor servicio, a través de personal calificado, certificaciones de calidad, inversiones en remodelaciones y equipamiento e infraestructura. Cabe señalar que, en la industria hotelera, los activos intangibles se convierten en la ventaja competitiva (Monfort, 2002), ya que aspectos como la imagen, los procesos organizacionales y el capital humano no se imitan tan fácilmente como las instalaciones físicas. Sin embargo, todos estos esfuerzos no se ven recompensados con mayores indices de ocupación.

Además, la industria hotelera es cíclica y sensible al comportamiento de la economía y a las circunstancias sociales y políticas (Fung et al., 2015), y presenta una alta sensibilidad a las condiciones del mercado, pues en periodos de crisis económica, estas erogaciones no se pueden reducir fácilmente (Alberca y Parte, 2013), entre otras razones, porque es necesario mantener los estándares de calidad para conservar la categorización de los hoteles en el mercado, acorde con las normas técnicas sectoriales que regulan el sector, aunque, como sostienen Dalir et al. (2020), uno de los principales impactos de la estacionalidad, tanto desde la demanda como de la oferta, es la variabilidad en la calidad del servicio. 
Para incrementar el número de visitantes a Medellín, los gobernantes han apostado por convertir la Ciudad en un destino de negocios y de eventos profesionales y académicos de ámbito internacional. Este se conoce como turismo MICE, sigla que se compone de las palabras en inglés: Meeting, Incentives, Congresses, Exhibition. Para Rangel y Rivero (2017), el principal beneficio de este tipo de turismo "es la posibilidad que ofrece de contribuir a desestacionalizar la oferta" (p. 238). El turismo de negocios se considera un sector de alto potencial en la actualidad; según el estudio MICE Travel Report 2018, se evidencia que los viajes MICE en América Latina han crecido de forma considerable (Pancorbo et al., 2019). En consecuencia, depende de la voluntad de las agremiaciones públicas y privadas la planificación de eventos con mayor frecuencia, que permitan extender la estadía y gasto del turista en la Ciudad.

De otro lado, también son importantes los aspectos internos, como la gestión del talento y el cuidado ambiental en las organizaciones, dos temáticas que actualmente se incorporan en la planeación estratégica de las empresas y que en los últimos años han ganado importancia en el mundo empresarial y académico. La convergencia de dichas temáticas implica la formación y orientación del talento humano hacia el cuidado de su relación personal y laboral, e induce a la creación de una política sustentable y al cuidado del medio ambiente en las organizaciones (Bermúdez-Tirado et al., 2016; Contreras y Peñaloza, 2018). En forma complementaria, Millán-García y Gómez-Díaz (2018) explican que los factores que producen la competitividad tienen que ver con los procesos y las condiciones del servicio, a la vez que se reconoce que estos varían en función de cada contexto turístico.

En este orden de ideas, este estudio se centró, específicamente, en el caso de una cadena hotelera, con presencia en el ámbito nacional, y cinco establecimientos de alojamiento en la ciudad de Medellín, que buscan atender diferentes segmentos del mercado, desde los viajeros de negocios hasta los usuarios que necesitan alojamiento para largas temporadas, pasando por los turistas que buscan descanso y relajación. La cadena, además de su página web, ofrece la posibilidad de reservas por medio de portales como Booking \& Expedia, los cuales permiten evaluar la experiencia del cliente, algo que también se intenta hacer directamente con los huéspedes que reservan por otros canales. Con el fin de mejorar la reputación en línea, los funcionarios de la cadena, monitorean constantemente los portales para conocer los comentarios, y cuando estos no son positivos se busca la forma de resarcir al cliente ofreciéndole descuentos u obsequios para visitas futuras. Esta gestión es muy importante, pues “la reputación en línea es crítica para definir la comunicación, la imagen y la estrategia de ventas en la industria hotelera" (Rodríguez-Díaz et al., 2019).

Para Ruizalba et al. (2015) la clave de la competitividad empresarial, en el sector hotelero, se relaciona con el capital humano, gestión del conocimiento y la orientación al marketing interno. Con base en estas corrientes, se realizó el diagnóstico de la cadena, para establecer las causas de los niveles poco satisfactorios de ocupación. Los resultados del diagnóstico fueron la base para diseñar algunas estrategias encaminadas a minimizar las amenazas y debilidades, y potenciar las fortalezas, de tal forma que se puedan aprovechar las oportunidades que ofrece el posicionamiento de la ciudad como uno de los destinos más visitados en el país, y que esto se traduzca en un incremento en los índices de ocupación. Teniendo en cuenta que se trata de una cadena tradicional, con un mercado diversificado, dado que Medellín tiene cinco hoteles con diferentes formatos, se considera que tanto el diagnóstico como las estrategias propuestas pueden ser de utilidad para el sector formal de hospedaje de Medellín y otras ciudades.

El presente estudio se desarrolló antes del inicio de la pandemia Covid-19; se debe tener en cuenta que el panorama del sector turístico y sus diferentes subsectores, cambiará drásticamente en el corto y mediano plazo, pues por temas asociados al confinamiento, aislamiento y distanciamiento social, se han cerrado las fronteras globales, lo que ha ocasionado un desplome con efectos negativos sin precedentes en toda la historia del turismo. 


\section{Metodología}

El trabajo se desarrolló con un enfoque cualitativo de tipo descriptivo. Para su desarrollo se realizó un análisis de la situación general que presenta el sector formal de hotelería en la ciudad de Medellín. Luego, se eligió una cadena hotelera, que fuese representativa de este sector, considerando el número de establecimientos en la ciudad y la variedad de formatos de alojamiento que se ofrece a través de ellos.

El estudio en la cadena se basó en un análisis interno de sus fortalezas y debilidades, para lo cual se tuvo en cuenta aspectos como la gestión humana, la innovación, procesos administrativos, mercadeo, internacionalización y gestión ambiental. También, se analizaron las oportunidades y amenazas que, básicamente, son las mismas para todo el sector de alojamiento formal.

La recolección de información para esta primera parte se basó tanto en fuentes primarias como secundarias. Se contó con la colaboración de varios funcionarios de la cadena de las áreas de mercadeo, ventas, administración y finanzas. Además, se examinaron los portales Trip Advisor, Booking y Expedia, con el fin de conocer las calificaciones y comentarios que han hecho los clientes del hotel. Como complemento a esta información se analizaron los estados financieros de la cadena.

También, se hizo revisión de prensa y revistas relacionadas con el sector turístico, tanto en el contexto local como nacional, para conocer las estadísticas de ocupación, llegadas de visitantes nacionales y extranjeros a la ciudad de Medellín, y políticas oficiales en relación con esta actividad económica. Las fuentes secundarias fueron, en su mayoría, artículos de revistas académicas y científicas relacionadas con el tema del turismo, en general, y del hospedaje, en particular.

En cuanto a las técnicas para la recolección de información, se privilegió la entrevista en profundidad con los empleados de la cadena y el análisis de contenido para la información documental y los datos de los portales mencionados.

Una vez identificadas las principales debilidades y amenazas, así como los aspectos más valorados por los clientes, se construyó el diagnóstico organizacional, con base en el cual se diseñaron estrategias encaminadas a mejorar el desempeño de la cadena, potenciar su imagen, y que esto, a su vez, mejore los índices de ocupación. Se propusieron 22 lineamientos de acción, que se agruparon en 8 categorías, así: mercadeo, medio ambiente, gestión humana, innovación, finanzas, procesos internos, internacionalización y parahotelería.

A continuación, se construyó un formulario tipo escala de Likert en el que se presentan los 22 lineamientos agrupados en las 8 categorías mencionadas, que permitió a los participantes elegir, para cada lineamiento, una opción de acuerdo con la lógica que se muestra en la tabla 1. Además, por cada lineamiento, el constructo permite que los respondientes puedan hacer comentarios. Esta herramienta se conoce como color insight, que se deriva de la prospectiva estratégica y consiste en la utilización de una escala, en la cual las opciones de opinión para los participantes se representan en una tabla de colores (Régnier, 1989); es un método recomendado por Durance y Godet (2011) para confrontar puntos de vista disímiles. Se seleccionó esta técnica de prospectiva participativa puesto que es una versión innovadora y un instrumento automatizado que sigue los principios del Ábaco de Régnier, método tradicional de consulta a expertos, destinado principalmente a reducir la incertidumbre en problemáticas concretas, el cual permite la recopilación y procesamiento de datos cualitativos. Además, los expertos tienen la facilidad de participar desde cualquier lugar a través de una computadora o dispositivo electrónico inteligente. El color insight utilizado es una versión multiplataforma en la nube de libre acceso y es compatible con cualquier navegador de internet; también se puede acceder a él desde dispositivos móviles. Para evaluar la validez del instrumento se aplicó a algunos funcionarios de la cadena y docentes universitarios de mercadeo, a quienes se les pidió responder y hacer comentarios tendientes a mejorar el instrumento. 
Una vez se tuvo el formulario definitivo, se procedió a su aplicación. La elección de los participantes se realizó con base en un muestreo mixto que comprende la mezcla entre la muestra de expertos y la muestra por conveniencia, que se basa en casos disponibles a los que se tiene acceso. Se estableció contacto con funcionarios de entidades relacionadas con el turismo y la hotelería, como la Subsecretaría de Medellín y Cotelco, docentes de universidades del área de administración, como la Universidad Nacional, el Politécnico Jaime Isaza Cadavid y algunos profesores que ofrecen asignaturas en el programa Administración de Empresas Turísticas del Colegio Mayor de Antioquia. También, respondieron el cuestionario funcionarios del área comercial de la cadena hotelera y de otros hoteles de la ciudad. En total, se obtuvieron 21 respuestas válidas

\section{Tabla 1}

Lógica del color

\begin{tabular}{lcc}
\hline \multicolumn{1}{c}{ Respuesta } & Color & Escala Likert \\
\hline No sé qué responder & & 0 \\
Completamente de acuerdo & & 1 \\
De acuerdo & & 2 \\
Diversos puntos de vista & & 3 \\
Desacuerdo & & 4 \\
Completamente en desacuerdo & & 5 \\
\hline
\end{tabular}

Nota: elaboración propia con base en las opciones de respuesta en Color Insight

Posteriormente, se evaluó la fiabilidad del instrumento, utilizando el Coeficiente Alfa de Cronbach, con un resultado de 0.90 , el cual es un coeficiente alto, por lo que se puede decir que el instrumento es fiable. Finalmente, se realizó el análisis de resultados con el apoyo del software Color Insight. Gracias a este instrumento, las respuestas son procesadas en tiempo real, permitiendo el procesamiento automático de las matrices según la escala cromática y las ponderaciones porcentuales para cada una de las categorías; el análisis se presenta en la sección de resultados.

Finalmente, en cuanto a las consideraciones éticas, el proyecto se desarrolló con base en buenas prácticas de investigación, cumpliendo los principios básicos de honestidad, responsabilidad y rigor, así como el respeto de la propiedad intelectual, con la adecuada citación de las fuentes consultadas.

Dado que no se indagó sobre aspectos personales de los participantes en el estudio, no se incluyó el consentimiento informado por escrito. De otro lado, teniendo en cuenta que se mencionan algunas debilidades de la cadena, no se divulga el nombre de la misma. 


\section{| Resultados}

\section{Evolución del sector hotelero en Medellín}

Es evidente el incremento de la oferta de establecimientos de alojamiento en la Ciudad, especialmente a partir de 2015, con la construcción de nuevos hoteles, tanto de las marcas existentes como con la entrada de cadenas internacionales: Blue Doors, Accor Hotels, Best Western, Hilton, Wyndham, Quinta Inn, Marriott, NH Hotels, City Express, Intercontinental, Selina, Decameron, Travelers, Atton, Viaggio y Click Clack. (ver tabla 2).

\section{Tabla 2}

\section{Oferta hotelera en la Ciudad de Medellín}

\begin{tabular}{ccccc}
\hline Año & 2015 & 2016 & 2017 & 2018 \\
\hline Hoteles & 96 & 176 & 197 & 205 \\
Habitaciones & 5411 & 7370 & 8628 & 9349 \\
Camas & 8073 & 12006 & 13870 & 14410 \\
\hline
\end{tabular}

Nota: elaboración propia con base en el informe de gestión 2018 y 2019 de Cotelco

Aunque no se conoce la cifra oficial de oferta hotelera en Medellín para 2019 (la entidad encargada de esta información es Cotelco, y no se ha publicado a la fecha), se cree que el número de habitaciones sigue en aumento, teniendo en cuenta que algunas cadenas hoteleras, como Estelar, iniciaron ese año operaciones con dos nuevos establecimientos en el Poblado: Estelar La Torre Suites, con una línea de negocio prime enfocada a largas estadías, y Estelar Square, dirigido al segmento de turistas que visitan la ciudad por motivos de ocio y esparcimiento, a través de cortas estadías con un presupuesto moderado. Así mismo, otras cadenas hoteleras recientemente iniciaron sus operaciones en el mercado: el hotel Novotel Medellín El Tesoro (Ex Atton) y el Class Suites. Además, se encuentran en fase de construcción nuevos proyectos como el Bluedoors York Luxury, también en el Poblado.

Como se explicó en el apartado anterior, con base en el diagnóstico, las opiniones de los expertos y la revisión de literatura, se construyeron los lineamientos de acción que deben tenerse en cuenta para mejorar la ocupación de la cadena. En la figura 1 se presenta la ruta metodológica y resultados. 
Figura 1

Ruta metodológica y resultados.

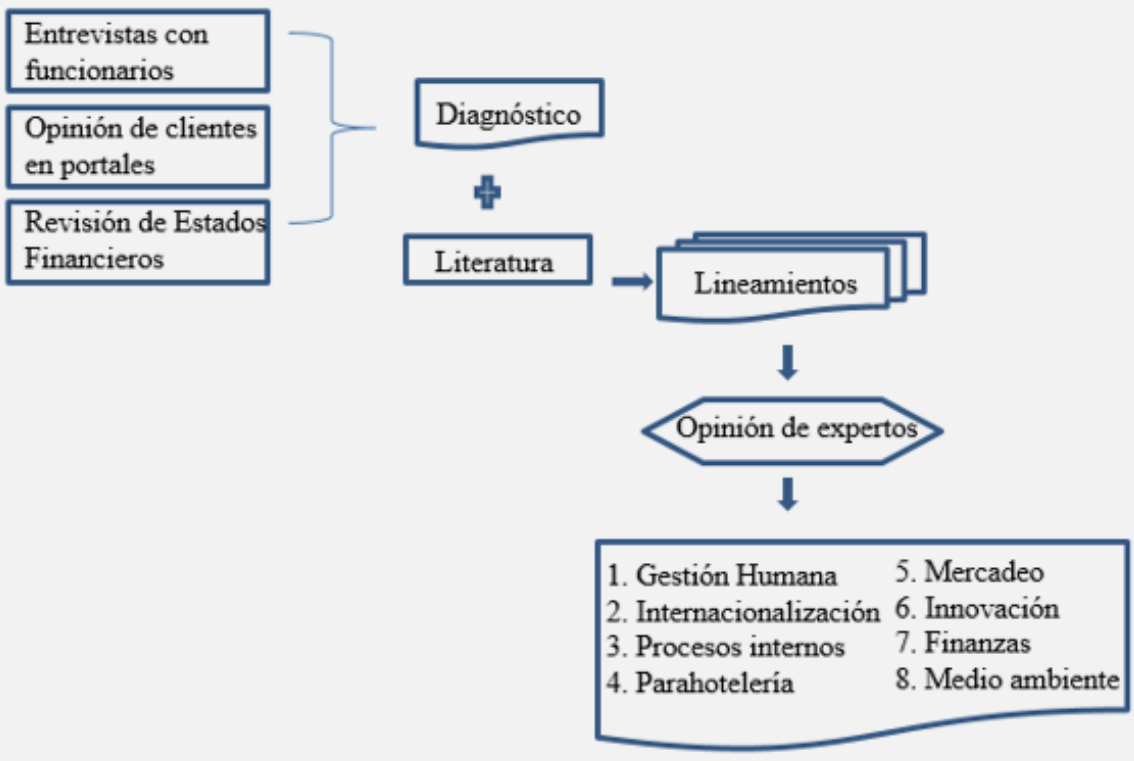

\section{Nota: elaboración propia.}

A continuación, se listan las categorías en el orden que fueron presentadas a los expertos a través del Color Insight. Para efectos del ejercicio investigativo la secuencia fue la siguiente: mercadeo, medio ambiente, gestión humana, innovación, finanzas, procesos internos, internacionalización y parahotelería. En cada una de ellas se desglosan los respectivos lineamientos de acción.

\section{Mercadeo}

Los lineamientos están orientados a regularizar la demanda, ya que es común que en la cadena se presente mayor ocupación entre lunes y viernes, que los fines de semana.

Alianzas operadores: se recomienda la creación de paquetes turísticos de diferente indole, en colaboración con otras empresas del sector turístico, como agencias de viaje, empresas de transporte y guías.

Bienestar: diversificar la oferta de servicios, especialmente los fines de semana cuando la ocupación es más baja. Se pueden diseñar planes especiales con descuentos para los huéspedes frecuentes, lo que, a su vez, incentivaría la fidelización, pero también atraería clientes nuevos si se aprovechan tendencias como turismo de compras, ocio, relajación, conciencia por la salud física, emocional y psíquica. 
Millenials: actualmente los jóvenes tanto del país como del exterior viajan más, convirtiéndose en un mercado atractivo. Hay que tener en cuenta que sus gustos y necesidades son diferentes a las de otros tipos de clientes.

Promoción: los hoteles pueden incluir dentro de sus servicios la celebración de fechas especiales como bodas y aniversarios. Estos pueden promocionarse a través de redes sociales y plataformas como Groupon. También, se pueden firmar acuerdos con empresas y fondos de empleados, ofreciendo tarifas especiales.

Otras acciones de mercadeo que se proponen para incrementar la ocupación son:

Alianzas competidores: cuidándose de incurrir en prácticas ilegales se pueden generar compromisos, entre los prestadores de servicios de alojamiento legales, de no mejorar las tarifas por debajo de los costos que implica la prestación de un servicio de calidad.

Cortesías: una forma de contribuir con los productores locales es la oferta de cortesías para los clientes, a partir de productos autóctonos, de calidad y que guarden sintonía con el cuidado de la salud y el medio ambiente,

Oferta: se sugiere revisar la segmentación de acuerdo con los formatos actuales de los establecimientos de la cadena. Por ejemplo, para los viajes de negocios, muchas empresas ya no están enviando a los altos ejecutivos, sino a los mandos medios, lo que requiere una oferta de servicios y tarifas diferentes.

\section{Medio ambiente:}

Gestión ambiental: realizar programas de responsabilidad social corporativa, que impactan tanto la competitividad como el desempeño, incluyendo popularidad, participación de mercado y actividades relacionadas con el talento humano (Mehrez, 2020). Aproximadamente el $85 \%$ de los viajeros del mundo, según Booking, están dispuestos a pagar un mayor precio por hospedarse en lugares amigables con el medio ambiente (Viva Air, 2018). En este sentido, la cadena debe propender porque sus acciones guarden relación con los principios del pacto global, los objetivos del desarrollo sostenible y el Global Reporting Initiative (GRI Standards), empezando, por ejemplo, con el uso de energías alternativas para aires acondicionados y calentadores de agua. Además, realizar campañas para promover la conciencia ambiental en los clientes, con actividades como: incentivar la reducción en el uso de empaques plásticos, dejando botellas de agua en vidrio en las habitaciones y proveer recipientes para la separación de los residuos sólidos.

Amenities: Se refiere a los detalles que obsequian los hoteles para facilitar la experiencia del cliente; comprende diversos utensilios como: jabones, crema de manos, enjuague bucal, lustra calzado, pañuelos, champú y acondicionador. Buscar proveedores que fabriquen este tipo de productos de manera ecoamigable y que sean de buena calidad, y que generen una buena experiencia para el cliente, con aromas y efectos sensoriales, que se conviertan en una de las fuentes de recordación del servicio. Para ser coherentes con la propuesta ambientalista, se recomienda utilizar únicamente dispensadores de jabones y champú, y suministrar otros amenities en caso de el huésped lo solicite.

\section{Gestión humana:}

Capacidades: en el diagnóstico se encontró que hay falta de motivación del personal, causada por altas cargas de trabajo y salarios no acordes con las funciones realizadas, lo que ocasiona una alta rotación, afectando a su vez, la curva de aprendizaje de los empleados. Para mejorar dicha motivación se recomiendan acciones como: equidad en términos de retribución salarial, pues se percibe un principio mercantil muy marcado al interior de la organización, crear programas de incentivos acordes con las funciones, el desempeño y la formación, ofrecer oportunidades para la capacitación, tanto formal como informal. 
Oportunidades: más allá del aspecto puramente económico, la contraprestación a la labor de los empleados debe comprender las oportunidades de desarrollo personal y profesional, condiciones agradables del sitio de trabajo, buen trato, reconocimiento de logros, planes de carrera al interior de la empresa y un esquema de contratación que ofrezca seguridad.

\section{Innovación:}

Velázquez (2019), refiriéndose a la innovación en la industria hotelera, afirma que las empresas tienen que crear diferentes modelos de negocios, modificar la gestión organizacional y aplicar mejores estándares de calidad para atraer nuevos mercados; para la autora, parte de esta innovación se basa en el uso de la tecnología. Para el caso de la cadena, se recomienda implementar tecnologías que faciliten la experiencia del cliente en los distintos momentos del servicio, que deben ser evaluadas de acuerdo con los requerimientos de los huéspedes y las posibilidades económicas. En este sentido, existen opciones como: los chatbots: asistentes virtuales que mejoran la interlocución con los usuarios, en relación con el servicio; Smart hotel room: consiste en la posibilidad que tiene el cliente, a través de un dispositivo móvil, de controlar ciertas condiciones de la habitación, como la temperatura del agua en la ducha y la iluminación, entre otras. Incluso hay posibilidades más sofisticadas como el Laboratorio de innovación Pop-Up Hotel, el cual consiste en alojamientos temporales que recrean experiencias que permiten al huésped activar todo su sistema sensorial.

Inteligencia artificial: se recomienda el uso de herramientas como la minería de datos, para analizar datos históricos en relación con el comportamiento de la demanda, que sirvan de base para estimar la ocupación futura de las habitaciones. Los modelos asociados a la analítica de datos proporcionan información valiosa que sirve como base para la toma de decisiones gerenciales.

\section{Finanzas:}

La cadena presenta un alto nivel de endeudamiento; así mismo, de acuerdo con el estado de situación financiera, se observa que maneja grandes niveles de efectivo. Se recomienda el pago de las obligaciones financieras más costosas, con el fin de disminuir los gastos financieros, teniendo en cuenta que la compañía mantiene en promedio un saldo final anual de efectivo y equivalentes, cercano a los 11 mil millones de pesos.

Endeudamiento: se recomienda a los propietarios de la cadena incrementar el capital, puesto que el endeudamiento ha crecido de forma considerable, mientras que los aportes de los socios se han mantenido invariables durante los últimos 5 años. Esto significa que la construcción de los nuevos establecimientos se ha financiado mayoritariamente con deuda, lo que expone a la compañía a un riesgo muy grande de dificultades en caso de una contracción de la demanda.

A continuación, se presentan los lineamientos orientados a mejorar las operaciones internas de la cadena, especialmente la centralización en los procesos que genera dificultades en las reservas y lentitud en otros servicios.

\section{Procesos internos:}

Descentralización administrativa: actualmente los procesos administrativos de la cadena se encuentran centralizados, lo que permite un mayor control por parte de la dirección, pero ocasiona demoras en operaciones como los pagos y devoluciones a clientes internos, externos y proveedores, en los que se genera gran incomodidad. Se recomienda reforzar con personal adicional aquellos procesos más administrativos, de tal forma que no se pierda el control, y descentralizar los más operativos, para garantizar un mejor servicio. 
Programas de fidelización: actualmente la cadena ofrece un programa de puntos que premia la fidelidad del cliente. Estos se acumulan por la utilización de los diferentes servicios y, de acuerdo con la cantidad, los puede cambiar por consumos o estadías. El programa ha tenido buena aceptación, pero debe ser más difundido.

\section{Internacionalización:}

Mercadeo de ciudad: se deben realizar acciones coordinadas entre el sector público y el privado tendientes a potenciar la Ciudad como un destino atractivo, tanto en el ámbito nacional como internacional, con campañas publicitarias que se transmitan en medios masivos de comunicación de los mercados objetivo.

Mercados internacionales: el mercado actual de la cadena es, mayoritariamente, nacional, en el que goza de muy buen prestigio. Con el incremento de extranjeros que llegan a la ciudad, hay una oportunidad para atraer este mercado. Una forma de empezar a trabajar en este aspecto es considerar la clasificación de los visitantes, de acuerdo con el país de procedencia, realizada por Procolombia, así: consolidación, oportunidad y reconocimiento. Con los mercados en consolidación se cuenta con conectividad aérea directa en el país, estos son: Reino Unido, Canadá, Francia, El Salvador, Guatemala y El Caribe. Por su parte, los mercados de oportunidad, a pesar de que no se cuenta con conectividad aérea directa, presentan una buena dinámica en la llegada de turistas, los cuales son: Turquía, Italia, Bélgica, Holanda, Suiza, Uruguay, Bolivia, China y Australia. Por último, se consideran los mercados en etapa de reconocimiento: Austria, Rusia, Corea del Sur, Emiratos Árabes, India, Indonesia, Japón y Singapur (Guerrero, 2017).

Lo anterior, requiere de acciones en varios frentes como: presentación de la página web en varios idiomas, atención del contact center, recepción y otros servicios a través de personal multilingüe. Así mismo, optimización en los motores de búsqueda, piezas publicitarias en medios extranjeros y alianzas con Online Travel Agencies de talla mundial.

\section{Parahotelería:}

Para enfrentar esta amenaza, se requiere el concurso del sector público y el privado, con acciones como el diseño y lanzamiento de campañas en las que se destaquen las ventajas asociadas a la hotelería formal: la seguridad, privacidad, confort, calidad y la importancia de la legalidad a través del buen uso de los impuestos.

Para conocer el impacto de cada uno de los lineamientos se recomienda la implementación de indicadores de gestión que permitan evaluar aspectos como: clima laboral, aumento en la ocupación, diminución de quejas y ampliación del nicho de mercado.

Evaluación de los lineamientos:

En la figura 2 se presenta la matriz general de resultados, de acuerdo con la lógica de color presentada en la tabla 1. Luego se muestra la figura 3 que contiene los resultados por ítem. Ambas figuras son construidas desde el software Color Insight. En dichas figuras se puede apreciar la escala codificada por colores; en las filas se encuentran los lineamientos, y en las columnas las respuestas ofrecidas por los expertos. En la matriz general de resultados es posible visualizar simultáneamente la posición de cada experto, para cada uno de los ítems planteados, mientras que la matriz por ítem aglutina las votaciones de todos los participantes, agrupando las respuestas por colores, de acuerdo con la escala cromática. 
Figura 2

\section{Matriz general de resultados}

Crear alianzas con agencias de viajes, empresas de transporte y guias turisticos, con el fin de diseriar paquetes turisticos en Medelin, y se genere una cultura de trabajo en red en pro el sector turistico.

Implementar alianzas estratégicas con los competidores del mercado hotelero tradicional, de tal forma que se evite la competencia directa y riesgos respecto a la canibalización de las marcas

Diseñar planes especiales para los fines de semana, con descuentos para los huéspedes frecuentes, con el fin de atraer nuevos mercados asociados al turismo de compras, leisure, salud, wellness.

Expansión hacia nuevos mercados por medio del posicionamiento SEO en los buscadores online, pautas publicitarias en periódicos internacionales y alianzas con Online Travel Agencies de talla mundial.

Inchuir al segmento de jóvenes y millenials de Colombia y el extranjero, ya que es un mercado atractivo que ahora excursiona más, a través de la adecuación del hotel de formato esencial a través de servicios de bajo costo y con buen servicio.

Diseñar una oferta de paquetes para celebraciones de aniversarios, cumpleaños, bodas, entre otros eventos

especiales, promocionándolos a través de redes sociales, fondos de empleados y plataformas como Groupon, con descuentos especiales para huéspedes frecuentes, compras anticipadas y cumpleaìeros.

Personalizar las cortesias ofrecidas a los buéspedes frecuentes con productos locales y autóctonos, de calidad y que guarden sintonia con el cuidado de la salud, a través de alianzas con marcas reconocidas a nivel local y nacional

Analizar la segmentación actual de los formatos de hoteles existentes, ya que hoy en dia las orgarizaciones no sólo envian de viaje a los ejecutivos altos sino a los mandos medios, dividiendo estratégicamente la oferta según las necesidades y caracteristicas de la demanda.

Emitir declaraciones que promuevan la conciencia medio ambiental en los clientes: manejo integral de residuos sólidos por medio de su separación (reciclables, orgánicos, ordinarios y peligrosos), uso de energjas alternativas para aires acondicionados y calentadores de agua.

Suscribir convenios con grandes firmas cosméticas que promuevan la conservación del medio ambiente para fortalecer el aprovisionamiento de amenities en las habitaciones, utilizando únicamente dispensadores de jabones y shampoo. Se suministrarán otros amenities en caso de que cada huésped lo solicite

Crear capacidades de planificación en gestión bumana que promuevan la retención, desarrollo y satisfacción de los empleados, bajo un marco de justicia en términos de retribución salarial. Logrando su motivación, reconocimiento a sus funciones y dignidad, se traducirá en un mejor servicio al cliente

Realizar una mayor difusión de los programas de fidelización, los cuales en su mayoria han sido bien aceptados por los clientes que los conocen en la actualidad

Medir la efectriviad de los lineamientos mediante el diserio de indicadores que evalúen el aumento en la ocupación, diminución de quejas, ampliación del nicho de mercado y clima laboral

Generar cooperación público-privada para promocionar el destino: marketing de ciudad, realizando vitrinas turisticas y campanas agresivas a nivel internacional, donde se promocione a Medellin como destino turistico, en conjunto con agencias de turismo receptivo y cadenas hoteleras.

Diseño de camparias publicitarias conjuntas con otros hoteles de la misma categoria, en redes sociales, donde se enseñen las ventajas asociadas a la hoteleria formal como la seguridad, privacidad, confort, calidad.

Promover la creación de un comité interestatutario donde participe el gobierno, los organismos de promoción del turismo y representantes del sector hotelero formal para implementar acciones concretas en contra de la parahoteleria

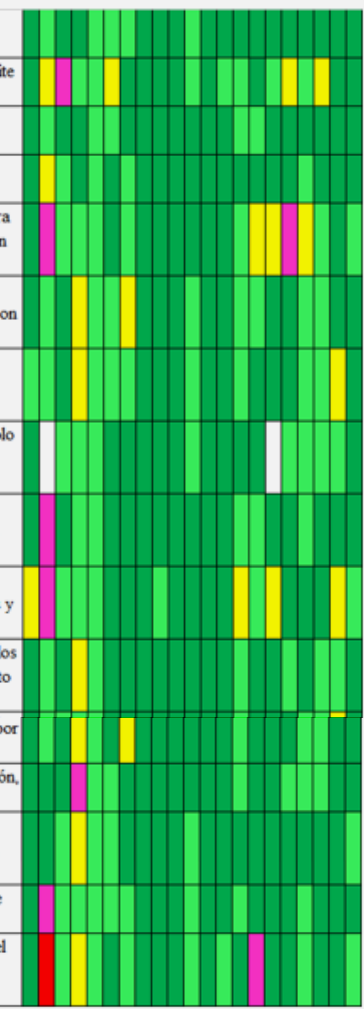

Nota: elaboración propia a través de los resultados del software Color Insight 
Figura 3

Matriz por ítem

Crear alianzas con agencias de viajes, empresas de transporte y guias turisticos, con el fin de diseñar paquetes turisticos en Medellin, y se genere una cultura de trabajo en red en pro el sector turistico.

Diseniar planes especiales para los fines de semana, con descuentos para los huéspedes frecuentes, con el fin de atraer nuevos mercados asociados al turismo de compras, leisure, salud, wellhess.

Expansión hacia nuevos mercados por medio del posicionamiento SEO en los buscadores online, pautas publicitarias en periódicos internacionales $y$ alianzas con Online Travel Agencies de talla mundial.

Generar cooperación público-privada para promocionar el destino: marketing de ciudad, realizando vitrinas turisticas y campatias agresivas a nivel intemacional, donde se promocione a Medelin como destino taristico, en conjunto con agencias de turismo receptivo y cadenas hoteleras.

Emitir declaraciones que promuevan la conciencia medio ambiental en los clientes: manejo integral de residuos sólidos por medio de su separación (reciclables, orgánicos, ordinarios y peligrosos), uso de energias altemativas para aires acondicionados $y$ calentadores de azua

Crear capacidades de planificación en gestión humana que promuevan la retención, desarrollo y satisfacción de los empleados, bajo un marco de justicia en téminos de retribución salarial. Logrando su motivación, reconocimiento a sus finciones y dignidad, se traducira en un mejor servicio al cliente

Realizar una mayor difusión de los programas de fidelización, los cuales en su mayoria han sido bien aceptados por los clientes que los conocen en la actualidad

Medir la efectividad de los lineamientos mediante el diseño de indicadores que evalúen el aumento en la ocupación. diminución de quejas, ampliación del nicho de mercado y clima laboral

Generar oportunidades de realización en los empleados, a través de adecuadas condiciones en el lugar de trabajo, el reconocimiento de logros, planes de carrera al interior de la empresa y un esquema de contratación que brinde seguridad Diseño de campanias publicitarias conjuntas con otros hoteles de la misma categoria, en redes sociales, donde se enseñen las ventajas asociadas a la hoteleria formal como la seguridad, privacidad, confort, calidad.

Descentralización de procesos administrativos que permitan la reducción de los reprocesos y por tanto se logre optimización de las tareas: como pagos y devoluciones a clientes internos, externos y proveedores, lo que permita facilitar una mejor experiencia para el cliente.

Diseñar una oferta de paquetes para celebraciones de aniversarios, cumpleanios, bodas, entre otros eventos especiales, promocionándolos a través de redes sociales, fondos de empleados y plataformas como Groupon, con descuentos especiales para huéspedes frecuentes, compras anticipadas y cumpleañeros.

Personalizar las cortesias ofrecidas a los huéspedes frecuentes con productos locales y autóctonos, de calidad y que guarden sintonia con el cuidado de la salud, a través de alianzas con mareas reconocidas a nivel local y nacional Introducir técnicas derivadas de la inteligencia artificial, para realizar proyecciones sobre el comportamiento del mercado. En este caso, podria potenciar la innovación del servicio al convertir patrones ocultos de conducta en iniciativas novedosas que motiven los clientes.

Evaluar la posibilidad de prepagar algunos pasivos con el fin de disminuir los gastos financieros, teniendo en cuenta que la compania mantiene en promedio un saldo final amual de efectivo y equivalentes de efectivo por valores superiores a los 11 mil millones de pesos en bancos

Promover la creación de un comité interestatutario donde participe el gobierno, los organismos de promoción del turismo y representantes del sector hotelero formal para implementar acciones concretas en contra de la parahoteleria Suscribir convenios con grandes firmas cosméticas que promuevan la conservación del medio ambiente para fortalecer el aprovisionamiento de amentites en las habitaciones, utitizando únicamente dispensadores de jabones y shampoo. Se suministrarán otros amentities en caso de que cada huésped lo solicite

Incorporar tecnologias que faciliten la experiencia del chente en los distintos momentos del servicio, como los Chat Bots, Smart Hotel Room y Pop-Up Hotel

Ante el incremento en el endeudamiento, se recomienda a los accionistas incrementar los aportes de capital, los cuales se han mantenido invariables durante los úttimos 5 atilos

Implementar alianzas estratégicas con los competidores del mercado hotelero tradicional, de tal forma que se evite la competencia directa y riesgos respecto a la canbalifización de las marcas

Analizar la segmentación actual de los formatos de hoteles existentes, ya que hoy en dia las organizaciones no sólo emvian de viaje a los ejecutivos altos sino a los mandos medios, dividiendo estratégicamente la oferta segin las necesidades y caracteristicas de la demanda.

Inchur al segmento de jóvenes y millenials de Colombia y el extranjero, ya que es un mercado atractivo que ahora

excursiona más, a través de la adecuación del hotel de formato esencial a través de servicios de bajo costo y con buen servicio.

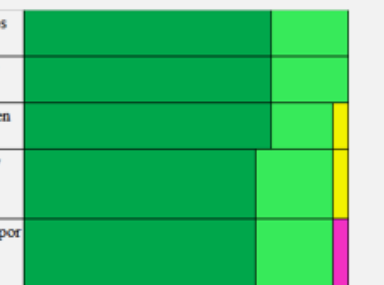

Nota: elaboración propia a través de los resultados del software Color Insight. 
En la tabla 3 se presenta un resumen de los resultados porcentuales para cada ítem, de acuerdo con las votaciones efectuadas por los expertos, siguiendo la lógica de respuestas del software Color Insight.

\section{Tabla 3}

\section{Resultados porcentuales}

\begin{tabular}{lccccccc}
\hline & $\begin{array}{c}\text { Completam. } \\
\text { de acuerdo }\end{array}$ & $\begin{array}{c}\text { De } \\
\text { acuerdo }\end{array}$ & $\begin{array}{c}\text { Diversos } \\
\text { puntos } \\
\text { de vista }\end{array}$ & Desacuerdo & $\begin{array}{c}\text { Completam. } \\
\text { en } \\
\text { desacuerdo }\end{array}$ & $\begin{array}{c}\text { No sé } \\
\text { qué } \\
\text { responder }\end{array}$ & Total \\
\hline Mercadeo & $58,3 \%$ & $31,5 \%$ & $7,1 \%$ & $1,8 \%$ & $0,0 \%$ & $1,2 \%$ & $100 \%$ \\
Medio Ambiente & $59,5 \%$ & $26,2 \%$ & $9,5 \%$ & $4,8 \%$ & $0,0 \%$ & $0,0 \%$ & $100 \%$ \\
Gestión Humana & $64,3 \%$ & $31,0 \%$ & $4,8 \%$ & $0,0 \%$ & $0,0 \%$ & $0,0 \%$ & $100 \%$ \\
Innovación & $50,0 \%$ & $38,1 \%$ & $7,1 \%$ & $0,0 \%$ & $0,0 \%$ & $4,8 \%$ & $100 \%$ \\
Finanzas & $42,9 \%$ & $42,9 \%$ & $11,9 \%$ & $0,0 \%$ & $0,0 \%$ & $2,4 \%$ & $100 \%$ \\
Procesos Internos & $61,9 \%$ & $30,2 \%$ & $6,3 \%$ & $1,6 \%$ & $0,0 \%$ & $0,0 \%$ & $100 \%$ \\
Internacionalización & $71,4 \%$ & $23,8 \%$ & $4,8 \%$ & $0,0 \%$ & $0,0 \%$ & $0,0 \%$ & $100 \%$ \\
Parahoteleria & $54,8 \%$ & $35,7 \%$ & $2,4 \%$ & $4,8 \%$ & $2,4 \%$ & $0,0 \%$ & $100 \%$ \\
\hline
\end{tabular}

Nota: elaboración propia con base en los resultados del Color Insight.

Como puede observarse en la tabla 3, los lineamientos con mayor votación, para los que la sumatoria de las opciones "Completamente de acuerdo" y "De acuerdo" estuvo por encima del 90 $\%$, fueron, en su orden: Gestión humana, Internacionalización, Procesos internos y Parahotelería. No obstante, todos tuvieron una votación superior al $85 \%$.

De acuerdo con la valoración de los expertos, el orden de los lineamientos es: i) Gestión humana; ii) Internacionalización; iii) Procesos internos; iv) Parahotelería; v) Mercadeo; vi) Innovación; vii) Finanzas, y viii) Medio ambiente.

\section{| Discusión}

Como lo expresa Monfort (2002), la competitividad de la industria hotelera está basada en gran medida en los activos intangibles, por lo que este tipo de activos tienen un gran peso en el posicionamiento de marca de las empresas dedicadas a esta actividad. El mejoramiento de imagen de Medellín como destino turístico y de negocios, sumado a los incentivos tributarios otorgados por el Gobierno, han llevado a que varias de las cadenas más reconocidas en el ámbito internacional estén haciendo presencia en la ciudad. Sin embargo, la ocupación hotelera en general no crece al mismo ritmo que el número de visitantes, lo que indica que hay otros factores que influyen en la decisión de los turistas en cuanto al alojamiento, como el económico, que los lleva a usar los portales como Aibnb, obviando las implicaciones de ilegalidad.

Sin embargo, no hay que buscar todas las causas de la baja ocupación hacia afuera, es necesario realizar también un análisis interno que permita identificar las debilidades que pueden estar agravando esta problemática. 
En un estudio similar, realizado por Santamaría y Cadrazco (2011), se utilizó la técnica Matriz de Inteligencia Hotelera para identificar las principales brechas entre las expectativas del cliente y sus percepciones, en relación con el servicio en el sector hotelero de Santiago de Tolú; el propósito del estudio fue proponer estrategias de mejoramiento de dichos hoteles. Este estudio también partió de un diagnóstico realizado a través de entrevistas en profundidad con directivos y empleados de los hoteles y algunos clientes.

Los resultados mostraron que el sector de hospedaje en Tolú tiene un desarrollo incipiente, pues no cuenta con personal suficientemente capacitado ni las condiciones para prestar un servicio de buena calidad (Santamaría y Cadrazco, 2011). Las principales falencias se encontraron en servicios básicos como la reservación, camareras, restaurante/cocina, relaciones públicas/publicidad, seguridad física, productos de turismo, seguridad industrial, mantenimiento, internet inalámbrico y cierre de cuentas. Así las cosas, las propuestas de mejora estuvieron orientadas al cubrimiento de estas necesidades, iniciando con procesos de capacitación, asociación de los hoteles y búsqueda del apoyo de entidades externas, como la Cámara de Comercio para el tema de publicidad, y la policía para los asuntos relacionados con seguridad.

Por su parte, la cadena hotelera tiene cubiertos todos estos requerimientos básicos, pues cuenta con un Departamento de Calidad que vela por el cumplimiento de políticas y estándares relacionados con la certificación y categorización de los hoteles, de acuerdo con las normas que regulan el sector de alojamiento. Las falencias se encuentran en otros aspectos como la falta de motivación del personal.

La motivación en el trabajo ha sido un tema de interés para las empresas, especialmente a partir de las investigaciones desarrolladas por Elton Mayo en Hawthorne. Los resultados de dicha investigación, con el apoyo de la psicología organizacional y la sociología industrial, han llevado al desarrollo de modelos que describen la conducta de los empleados como el resultado de una combinación de factores, entre los cuales el económico no siempre es el principal (Marulanda-Valencia et al., 2014).

Teniendo en cuenta que los hoteles operan 24 horas los 7 días de la semana, y no cierran sus puertas al público en ninguna fecha durante los 365 días del año, es probable que se requiera que los colaboradores trabajen horas extras en cualquier momento, ante un aumento repentino en el volumen de trabajo o la ausencia de algún empleado. En ciertos casos, esta situación genera desmotivación, principalmente cuando se trata de fechas especiales como, por ejemplo, el día de la madre/padre, Semana Santa o la temporada decembrina, entre otras. Esto puede convertirse en una fuente de pérdida de clientes, pues una persona que no se siente satisfecha en su trabajo, difícilmente podrá ofrecer un buen servicio. De hecho, esta puede ser la causa de las dificultades que manifiestan los clientes de la cadena, como reprocesos y demoras en la atención.

Otro factor que es cada vez más imperativo en las organizaciones, es el cuidado del medio ambiente. Posiblemente en el medio aún falta adquirir mucha conciencia sobre este aspecto, pero para muchos viajeros este es un factor muy influyente en sus decisiones de consumo, y cada vez más están en busca de “hospedajes verdes” (Bashir et al., 2019; Velázquez 2019). Además, para autores como Molina-Azorín et al. (2015), la gestión de calidad y la gestión ambiental en los hoteles permiten mejorar la ventaja competitiva, en términos de costos y diferenciación. 
Es así que, en un estudio realizado con hoteles mallorquines, Razumova et al. (2015) encontraron que innovaciones de tipo ambiental tienen potencial de ahorro de costos, además la categoría del hotel está asociada con innovaciones que contribuyen a la calidad del mismo, como el tratamiento de aguas residuales, aislamiento de ruido y mejoras en el impacto visual. Dentro de los lineamientos propuestos para la cadena hotelera, y que fueron bien calificados por los expertos, se propone, entre otros, el uso de energías alternativas, las cuales, además del beneficio ambiental, con el tiempo también significarán un ahorro de costos.

Podría decirse que estos dos aspectos: personal y medio ambiente, son los más importantes, ya que una buena gestión de estos, a través de innovaciones, puede influir favorablemente en otros aspectos como las finanzas, los procesos internos y la internacionalización de la cadena, ya que esto mejorará su imagen y se convertirá en una opción de hospedaje atractiva para los visitantes extranjeros.

Para abordar el mercado de extranjeros, la cadena debe prepararse ofreciendo capacitación a sus empleados en el manejo de otros idiomas, así como en temas culturales y de geopolítica. En relación con la internacionalización, D’ Meza et al. (2016) afirman que la tecnología de las cadenas hoteleras, de los países desarrollados, les permite llegar con ventajas a los países en desarrollo, en perjuicio del crecimiento de los grupos hoteleros nacionales. Por lo anterior, recomiendan potenciar las marcas hoteleras de estos países a través del crecimiento y calidad de la oferta. Además, priorizar las alianzas con agencias de viajes y líneas aéreas procedentes de los mercados emisores, incluso con participación accionaria. Otra forma de fortalecer la imagen es a través del uso de los portales especializados como Booking y Expedia, no solo para el ofrecimiento de los servicios, sino para evaluar la experiencia del cliente, cuando la reserva ha sido efectiva.

En el caso actual, también es primordial la acción de los organismos encargados de promover la ciudad como un destino atractivo, tanto para el turismo como para el desarrollo de eventos comerciales y académicos. Históricamente, se ha evidenciado que los agentes del sector privado consideran que una acción vital para el desarrollo del turismo, es que el Estado se involucre más, a nivel político y económico, con el sector y que se materialicen avances en materia de promoción turística (Aranda y Martínez, 2017). Para este propósito es necesaria la confluencia del sector público, liderado por la Subsecretaría de Turismo; el sector mixto, a través de la Fundación Greater Medellín Convention y Visitors Bureau, entidad que se encarga de promocionar la ciudad y el departamento en mercados nacionales e internacionales; y el sector privado, a través de las directivas de los hoteles y las agremiaciones como Cotelco.

Otro aspecto que contribuye a mejorar el posicionamiento de un destino, es la actitud de los residentes hacia los visitantes. En general, los habitantes de Medellín se consideran acogedores, y los turistas, tanto extranjeros como nacionales, resaltan este aspecto como una de las fortalezas de la ciudad; esto hace parte de lo que Medeiros y Da Costa (2019) consideran como el contexto regional. Para los autores, en relación con el turismo, es necesario tener en cuenta los intereses de los habitantes del lugar, ya que esta actividad afecta directamente su lugar de residencia. Estos aprendizajes contribuyen a la acumulación de capital físico, humano y social que forma la base de la capacidad de atracción del turismo, la cual, de acuerdo con Cirer-Costa (2020) es necesaria para sobrevivir frente a las conmociones externas. 
La presente investigación se diferencia de otras similares en varios aspectos. Se llevó a cabo en dos fases, siendo la primera el diagnóstico de la cadena, para la cual se utilizaron tanto fuentes primarias como secundarias; se destaca dentro de las primarias la participación de empleados de la misma organización y la revisión de opiniones de los clientes. Este diagnóstico sirvió de base para la formulación de propuestas tendientes a mejorar la ocupación hotelera. La segunda fase consistió en la validación de dichas propuestas, lo cual se realizó con el apoyo del Color Insight, herramienta que permite el estudio de fenómenos en el mediano y largo plazo, los cuales en la Ciudad y en el sector hotelero aún se encuentran en una fase embrionaria; además, para mayor confiabilidad de los resultados, se buscó el apoyo de los sectores académico, sectorial y comercial. Todos estos aspectos constituyen el valor agregado, y de esta forma se presenta una visión innovadora y colectiva del futuro, así como el diseño de acciones para ejecutarse en el presente, con el fin de llegar al escenario deseado.

Desde el punto de vista práctico, la cadena puede empezar por implementar estrategias que no requieran grandes inversiones de dinero. Así, para mejorar la motivación se pueden ofrecer incentivos en alojamiento, alimentación, celebración de eventos y banquetes, entre otros servicios que pueda prestar el hotel, de acuerdo con su capacidad ociosa; además, se pueden diseñar programas que premien a los empleados por sugerencias o proyectos pensados para mejorar los procesos y resultados de la compañía. También, se pueden reforzar las campañas ambientales con avisos en las habitaciones, ascensores y áreas comunes, así como algunos cambios menores, como permitir al cliente decidir la periodicidad del cambio de sábanas y toallas.

\section{| Conclusiones}

El incentivo tributario ofrecido por el Gobierno colombiano para motivar la entrada de nuevas marcas de hoteles al país y la construcción de nuevos establecimientos para las ya existentes, generó una oferta de camas y habitaciones que, no obstante, el crecimiento en el número de visitantes, es difícil de ocupar al $100 \%$.

Esta situación se ha convertido en un reto para los directivos de los hoteles, quienes deben diseñar estrategias de diferenciación o de costos, que permitan mantener unos niveles de ocupación por encima del punto de equilibrio. Para ello, es necesario realizar un diagnóstico sobre la situación interna, a la vez que monitorear el ambiente externo para identificar amenazas y oportunidades.

En cuanto a los factores internos, hay que tener en cuenta que el sector de acomodación es especialmente sensible a la percepción del cliente, ya que se trata de un servicio compuesto por una gran cantidad de variables como la limpieza, la comodidad, la seguridad, el contacto, la agilidad, la alimentación, entre otros. Por esto es tan importante la motivación del personal, ya que los empleados son los que tienen contacto directo con el usuario y de ellos depende, en gran parte, la calidad en la atención.

Así mismo, la gestión ambiental se está convirtiendo en una variable que juega un papel importante en la percepción de la imagen corporativa, no solo como parte de la responsabilidad social organizacional, sino por su contribución a la conciencia sobre la importancia del buen uso de recursos como el agua y la energía. A su vez, con ideas innovadoras, esto puede convertirse para la empresa en una disminución de los costos de operación y una forma de atraer nuevos clientes. 
La tecnología, como parte de la innovación, permite obtener realimentación, en relación con la experiencia de los usuarios, que se puede potenciar generando una comunicación bidireccional con estos, con beneficios como: oportunidad para promocionar nuevos servicios, fidelización y reputación en línea.

El desarrollo de estas estrategias requiere que la cadena se fortalezca financieramente. Se recomienda un incremento del capital social que permita disminuir el endeudamiento, especialmente el de largo plazo que suele ser más costoso. Además, se disminuye el riesgo al que está expuesta la organización por tener comprometido un gran porcentaje de sus ingresos en el servicio a la deuda.

En relación con los factores externos, los lineamientos más valorados fueron internacionalización y mercadeo de ciudad. En el primero es muy importante la acción de la cadena, que debe contar con fuentes de información y personal capacitado, no solo en manejo de otros idiomas, sino también en aspectos culturales, económicos, sociales y políticos de los países de origen de los visitantes más frecuentes a la Ciudad, además de tener en cuenta los segmentos priorizados por Procolombia. Una forma conveniente de concretar estas propuestas es la creación de un área dentro de la cadena o por lo menos el nombramiento de un empleado que se dedique exclusivamente a este asunto, por ejemplo, monitoreando la oferta de ferias internacionales y programando la participación en ellas; además, apoyando la gestión de la contratación y/o capacitación del personal especializado, diseño de la página web y de la publicidad.

Para enfrentar la amenaza de la hotelería paralela, se requiere una vez más la acción conjunta del sector público y privado, pues son muchos los sectores perjudicados. El primero es, obviamente, el hotelero que no puede competir con las bajas tarifas que se ofrecen en los hospedajes ilegales. Para las autoridades de la Ciudad el perjuicio se da en varias vías: de un lado, la disminución en el recaudo de impuestos, y de otro lado, quizá la más urgente de conjurar, la dificultad para controlar los hechos delictivos que se pueden cometer en esos sitios, como la distribución y consumo de alucinógenos y la prostitución de menores de edad.

Finalmente, se reitera que el estudio se concluyó en un periodo con antelación al inicio de la pandemia Covid-19, y que, debido a la crisis coyuntural derivada por este fenómeno, se modificarán los escenarios sucesivos del sector turístico, pues se trata de una crisis sin precedentes que restringe los desplazamientos de las personas, afectando negativamente a los prestadores de servicios turísticos en toda su cadena de valor. El reto ahora será la reactivación y fortalecimiento del turismo una vez superada la condición de pandemia.

\section{| Referencias}

Alberca, P., y Parte, L. (2013). Evaluación de la eficiencia y la productividad en el sector hotelero español: un análisis regional. Investigaciones Europeas de Dirección y Economía de la Empresa, 19(2), 102-111. https://doi.org/10.1016/j.iedee.2012.10.004

Aranda, M. L., y Martínez, C. P. (2017). La promoción turística privada en la España del primer tercio del siglo XX: los Sindicatos de Iniciativa y Turismo. Investigaciones de Historia Económica, 15(1), 38-46. https://doi.org/10.1016/j.ihe.2017.10.001

Bashir, S., GhaniKhwaja, M., Turi, J., \& Toheed, H. (2019). Extension of planned behavioral theory to consumer behaviors in green hotel. Heliyon 5(12), 1-8. https://doi.org/10.1016/j.heliyon.2019.e02974

Bermúdez-Tirado, S., Ramírez-Hoyos, M. A., \& Jaramillo-Arias, A. (2016). Preferencias en el uso del tiempo libre de los estudiantes universitarios con enfoque en el sector turismo. Revista CEA, 2(4), 75-88. 
Cirer-Costa, J. (2020). Economic and social resilience accounts for the recovery of Ibiza's tourism sector. Tourism Geographies, 1-22. https://doi.org/10.1080/14616688.2020.1722214

Contreras, S., y Peñaloza, L. (2018). El talento humano y el cuidado ambiental en la industria hotelera. Turismo y Sociedad, 23, 85-100.

Cotelco Antioquia. (2018). Informe de Gestión 2018, Asamblea Ordinaria de Afiliados 2018, Cotelco Capítulo Antioquia-Chocó. https://drive.google.com/file/d/18aE7Gd2kPNBRUxhSjDXSJGOVLGIiZQNx/view

Cotelco Antioquia. (2019). Informe de Gestión 2019, Asamblea General Ordinaria Afiliados, Cotelco Capítulo Antioquia. https://drive.google.com/file/d/1E1SwcvgWxpuExT7H-gZJsb9e_pZ-SaNk/view

D’ Meza, G., Zaldivar, M., \& Martín, R. (2016). La expansión internacional de la industria hotelera de los países desarrollados como opción estratégica para los países subdesarrollados. Economía y Desarrollo, 157(2), 23-38.

Dalir, S., Mahamadaminov, A., \& Olaya, H. (2020). Airbnb and Taxation: Developing a Seasonal Tax System. Tourism Economics, 1-14. https://doi.org/10.1177/1354816620904894

Departamento Administrativo Nacional de Estadística -DANE-. (2019). Boletín Técnico, principales indicadores del mercado laboral julio de 2019. https://www.dane.gov.co/files/investigaciones/boletines/ech/ech/bol_empleo_jul_19.pdf

De Duque, R., Isabel, R., Leguizamon, M., y Herrera, B. (2010). Un modelo de intervención para la hotelería no formal como actor para la competitividad y sostenibilidad del destino turístico. Anuario Turismo y Sociedad, 11, 201-218.

Duque, J. S. (2018). Incentivo tributario, el fin de una época. Hospitalidad y Negocios, (41), 16-20.

Durance, M. G., y Godet, M. (2011). La prospectiva estratégica para las empresas y los territorios. Dunod.

Fung C., King E. M., \& Kulendran N. (2015). Estimating Future Room Occupancy Fluctuations to Optimize Hotel Revenues. Journal of Travel \& Tourism Marketing, 32(7), 870-885. https://doi.org/10.1080/10548408.2015.1063827

González-Posada, D. M., y Reyes-Bedoya, N. (2019). Herramientas de gestión al alcance: caso red de hostales de la ciudad de Medellín. Revista CEA, 5(9), 113-129.

Guerrero, J. (2017). Perspectivas 2017, un año de dificultades y retos. Hospitalidad y Negocios, (29), 11.

Marulanda-Valencia, F. A., Montoya-Restrepo, I. A., y Vélez-Restrepo, J. M. (2014). Teorías motivacionales en el estudio del emprendimiento. Pensamiento \& Gestión, (36), 206-238.

Matiz, J., Quiroga, S., Isaza, S., Rojas, N. M., y Rodríguez, H. A. R. (2011). Turbulencia empresarial en Colombia: el caso del sector hotelero. Documentos de investigación, 102, 1-46.

Medeiros, F. G., y Da Costa, F. J. (2019). Uma proposta de visualização do sistema agregado de marketing turístico. Estudios Gerenciales, 35(152), 237-248. https://doi.org/10.18046/j.estger.2019.152.3262

Mehrez, A. (2020). Social responsibility and competitiveness in hotels: The role of customer loyalty. Management Science Letters, 10(8), 1797-1802. https://doi.org/10.5267/j.msl.2019.12.039

Millán-García, C., y Gómez-Díaz, M. (2018). Factores e indicadores de competitividad hotelera. Compendium, 21(40), 1-20

Ministerio de Comercio Industria y Turismo -MINCIT-. (2018). Plan Sectorial de Turismo 2018-2022, Turismo: El propósito que nos une. http://www.mincit.gov.co/CMSPages/GetFile.aspx?guid=2ca4ebd7-1acd-44f9-9978-4c826bab5013

Ministro del Interior y de Justicia. (2003). Decreto 2755, Por medio del cual se reglamenta el artículo 207-2 del Estatuto Tributario. https://minciencias.gov.co/sites/default/files/upload/reglamentacion/decreto-2755-2003.pdf 
Molina-Azorín, J., Tarí, J., Pereira-Moliner, J., López-Gamero, D., \& Pertusa-Ortega, E. (2015). The effects of quality and environmental management on competitive advantage: A mixed methods study in the hotel industry. Tourism Management, 50, 41-54. https://doi.org/10.1016/j.tourman.2015.01.008

Monfort, V. M. (2002). Estrategia competitiva y desempeño en la industria hotelera costera: evidencias empíricas en Benidorm y Peñíscola. Cuadernos de Turismo, 10, 7-22.

Monsalve, C., y Hernández, S. I. (2015). Gestión de la calidad del servicio en la hotelería como elemento clave en el desarrollo de destinos turísticos sostenibles: caso Bucaramanga. Revista EAN, 78, 160-173.

Moreno-Izquierdoa, L., Rubia-Serranob, A., Perles-Ribesa, J., Ramón-Rodrígueza, A., \& Such-Devesa, M. (2020). Determining Factors in the Choice of Prices of Tourist Rental Accommodation. New Evidence Using the Quantile Regression Approach. Tourism Management Perspectives, 33, 1-11. https://doi.org/10.1016/j.tmp.2019.100632

Pancorbo, J. A., Cuenca, C., Fabian, G., Rodríguez, Y., Vega, V., Ricardo, L., \& Emilia, S. (2019). Retos y estrategias para el desarrollo del turismo de negocios en ciudades ecuatorianas. Revista San Gregorio, (35), 29-50. http://dx.doi.org/10.36097/rsan.v1i35.1123

Presidencia de la República de Colombia. (2016). Decreto 463, por el cual se modifica el Decreto 2755 de 2003, modificado por el Decreto 920 de 2009. https://dapre.presidencia.gov.co/normativa/normativa/DECRETO\%20463\%20DEL\%2016\%20DE\%20MARZO\%20DE\%202016.pdf

Rangel, C. R., y Rivero, M. S. (2017). Análisis de las necesidades formativas en el ámbito del turismo MICE en Extremadura: propuesta de nuevas competencias profesionales en Las tecnologías de información y comunicación para la innovación y el desarrollo. Alexandria Library Publishing House.

Razumova, M., Lozano-Ibáñez J., y Rey-Maquieira, J. (2015). Drivers of environmental innovation in Majorcan hotels. Journal of Sustainable Tourism, 23(10), 1529-1549. https://doi.org/10.1080/09669582.2015.1062016

Régnier F. (1989). Annoncer la couleur: pour une approche nuancée du consensus. Institut de Métrologie Qualitative, Pierre Belfond.

Rodríguez-Díaz, M., Alonso-González, R., Rodríguez-Voltes, C., \& Rodríguez-Voltes, A. (2019). A Rating of the Online Reputation Balance in Lodgings. Administrative Sciences, 9(58), 1-18. https://doi.org/10.3390/admsci9030058

Ruizalba, J. L., Vallespín, M., \& Pérez-Aranda, J. (2015). Gestión del conocimiento y orientación al marketing interno en el desarrollo de ventajas competitivas en el sector hotelero. Investigaciones Europeas de Dirección y Economía de la empresa, 21(2), 84-92. https://doi.org/10.1016/j.iedee.2014.03.001

Santamaría, A., y Cadrazco, W. (2011). Matriz de Inteligencia Hotelera - MIH Una propuesta para el mejoramiento de la calidad en la prestación del servicio hotelero. Pensamiento \& Gestión, 31, 211-246.

Sistema de Indicadores Turísticos de Medellín y Antioquia -SITUR-. (2020). Ingreso anual de extranjeros y colombianos por punto migratorio José María Córdova. http://situr.gov.co/estadisticas/DfMelngresoPasajerosAnual/general

Velázquez, J. A. (2019). Gestión de la innovación para la sustentabilidad de las empresas de hospedaje. Rosa dos Ventos - Turismo e Hospitalidade, 11(4), 819-831. http://dx.doi.org/10.18226/21789061.v11i4p819

Viva Air. (septiembre, 2018). Tendencias de Turismo. Revista Viva Air, (75), 62-63.

Zervas, G., Proserpio, D., y Byers, J. (2017). The rise of the sharing economy: Estimating the impact of Airbnb on the hotel industry. Journal of marketing research, 687-705. https//doi.org/10.1509/jmr.15.0204 